\title{
Feedback Control of Linear Quantum Optical Systems
}

\author{
Hu Zhang, Guofeng Zhang, Daoyi Dong, Bo Huang, H.W.J. Lee
}

\begin{abstract}
This paper studies feedback quantum control of a class of linear quantum optical systems by means of squeezing and phase modulation. A new quadrature representation of such systems is introduced, which includes explicitly phase modulation of light fields involved. Some fundamental relations are derived in terms of the new quadrature representation. The coherent quantum LQG control studied in [17] is re-investigated base on controller parametrization and a two-stage optimization approach. Examples are given to illustrate the effectiveness of the results in the paper.

Index Terms-quantum optics, LQG control, squeezing, phase modulation, optimization
\end{abstract}

\section{INTRODUCTION}

Feedback has been an effective mechanism in complex systems due to its capability to provide efficient and reliable performance despite ever increasing complexity and miniaturization. Recent advances in quantum- and nanotechnology have presented a great impetus for research in the area of quantum feedback control systems. In fact, a wide variety of control techniques have been successfully applied to experiments in the fields of quantum photonics, Bose-Einstein condensates, quantum computation, quantum information, quantum metrology, and so on. In recent years quantum optics is undergone considerable development in part because it provides building blocks for constructing quantum computing and communication devices to realize the dream of quantum information technology. The basic ingredients of a quantum optical system are cavities, beam splitters, modulators, phase shifters, amplifiers, fibres, and photodetectors, etc. The aim of control is to manipulate effectively such a system to perform prescribed tasks such as protecting the system from undesirable injected laser signals [14]. Quantum feedback control involves the interconnection of two systems (the quantum plant and the controller) in such a way that the plant-controller system achieves desired performance specifications. The controller itself may or may not be a quantum system. In the later case, the controller is

This work was partially supported by the National Natural Science Foundation of China under Grant 60804015, RGC PolyU 5203/10E, and the Australian Research Council (DP1095540).

H. Zhang, G. Zhang, and B. Huang are with the School of Electronic Engineering, University of Electronic Science and Technology of China, Chengdu, Sichuan, China 610054, henry6244906ahotmail.com, gfzhang@uestc.edu.cn, huangbwly@126.com. G. Zhang is also with the School of Engineering and Computer Science, the Australian National University, Canberra, ACT, 0200, Australia.

D Dong is with the School of Engineering and Information Technology, University of New South Wales at the Australian Defence Force Academy, Canberra, ACT 2600, Australia, daoyidong@gmail. com.

H.W.J. Lee is the Department of Applied Mathematics, the Hong Kong Polytechnic University, Hong Kong, China majlee@polyu. edu. hk. a classical system (often implemented by means of digital or analog circuits) and the feedback loop involves measurement; hence this is called measurement-based feedback.

Measurement-based feedback control of quantum optical systems has undergone a process from physical modeling to statistical inference and then to feedback control. A quantum stochastic control problem is addressed by Belavkin [2], where the problem is reduced to optimal control of a quantum system by a discrete control input sequence in terms of the initial system state and a discretely measured output sequence. By approximating field noise as white noise and assuming weakly coupling of field-cavity coupling, a linear quantum stochastic systems modeling framework in terms of a set of quantum stochastic differential equations (QSDEs) was ingeniously proposed by Hudson and Parthasarathy [11] in 1984. Interested reader may refer to [2], [4], [20], [6], [18], and [15] for excellent discussions on measurementbased feedback control and their applications to a broad range of quantum optical systems.

Besides measurement-based feedback control, there is another avenue to quantum feedback control. In this scenario, the controller itself is also quantum. The quantum controller exchanges quantum information with the quantum plant in the feedback loop. This quantum feedback mechanism is known as coherent feedback control. Quantum information may flow directionally as a (possibly non-commutative) signal (such as a quantum optical electromagnetic field or an injected laser), or directly via a bidirectional physical coupling. For convenience, the former is called field-mediated coherent feedback while the latter direct coupling. Coherent feedback control has been undergoing an extensive study in recent years [21], [8], [12], [14], [17], [16], [9], [13].

The purpose of this paper is to study quantum optical systems including squeezing components and phase modulators [1] from a control theoretical perspective, aiming at providing systematic control techniques for a wide spectrum of applications, e.g., LQG feedback control of quantum optical systems.

The rest of the paper is organized as follows. In section II, the class of linear quantum optical systems of interest is presented. Section III presents the set-up of closed-loop systems. Section IV studies coherent quantum LQG control. Section V concludes the paper.

Finally, some words for notation.

Notation. Let $i=\sqrt{-1}$ be the imaginary unit. Given a column vector of operators $x=\left[\begin{array}{lll}x_{1} & \cdots & x_{m}\end{array}\right]^{T}$ where $m$ is a positive integer, define $x^{\#}=\left[\begin{array}{lll}x_{1}^{*} & \cdots & x_{m}^{*}\end{array}\right]^{T}$, where the asterisk $*$ indicates Hilbert space adjoint or complex conjugation. Furthermore, define the doubled-up column 
vector to be $\breve{x}=\left[\begin{array}{ll}x^{T} & \left(x^{\#}\right)^{T}\end{array}\right]^{T}$. The matrix case can be defined analogously. The symbol $\operatorname{diag}_{n}(M)$ is a block diagonal matrix where the square matrix $M$ appears $n$ times as a diagonal block. Given two matrices $U, V \in \mathbb{C}^{r \times k}$, a doubled-up matrix $\Delta(U, V)$ is defined as $\Delta(U, V):=$ $\left[\begin{array}{llll}U & V ; & V^{\#} & U^{\#}\end{array}\right]$. Let $I_{n}$ be an identity matrix. Define $J_{n}=\left[\begin{array}{cccc}0 & I_{n} ; & -I_{n} & 0\end{array}\right]$ and $\Psi_{n}=\operatorname{diag}\left(I_{n},-I_{n}\right)$. (The subscript " $n$ " is always omitted.) Then for a matrix $X \in$ $\mathbb{C}^{2 n \times 2 m}$, define $X^{b}:=\Psi_{m} X^{\dagger} \Psi_{n}$. Finally, the norm symbol $\|\cdot\|$ represents the largest singular value for a constant matrix.

\section{Linear QUANTUM OPTICAL SYSTEMS WITH SQUEEZING COMPONENTS}

\section{A. Boson Fields}

The component of an ( $m$-channel) electromagnetic field in free space is described by a vector $b_{i n}(t)=$ $\left[b_{i n, 1}(t), \ldots, b_{i n, m}(t)\right]^{T}$, which satisfy the singular commutation relations

$$
\begin{gathered}
{\left[b_{i n, j}(t), b_{i n, k}^{*}\left(t^{\prime}\right)\right]=\delta_{j k} \delta\left(t-t^{\prime}\right),} \\
{\left[b_{i n, j}(t), b_{i n, k}\left(t^{\prime}\right)\right]=\left[b_{i n, j}^{*}(t), b_{i n, k}^{*}\left(t^{\prime}\right)\right]=0 .}
\end{gathered}
$$

The operators $b_{i n, j}(t)$ may be regarded as a quantum stochastic process; in the case where the field is in the vacuum state, this process is quantum white noise. The integrated processes $B_{i n, j}(t)=\int_{0}^{t} b_{i n, j}(\tau) d \tau$ are quantum Wiener processes [6, section 5.3.5], which may be used to define quantum stochastic integrals, with associated non-zero Ito product $d B_{i n, j}(t) d B_{i n, k}^{*}(t)=\delta_{j k} d t$.

\section{B. Open Quantum Optical Systems}

An open quantum optical system $G$ is a collection of $n$ quantum harmonic oscillators interacting with $m$ boson fields $b_{i n}(t)$. When there is no scattering among $b_{i n}(t)$, such a system can be parameterized by a triple $\left(I_{m}, L, H\right)$. In this triple, the vector operator $L$ is defined as

$$
L=C_{-} a+C_{+} a^{\#},
$$

where $C_{-}$and $C_{+} \in \mathbb{C}^{m \times n}$ and $a=\left[a_{1}, \ldots, a_{n}\right]^{T}$ with $a_{j}$ being the annihilation operator of the $j$ th quantum harmonic oscillator satisfying the canonical commutation relations $\left[a_{j}, a_{k}^{*}\right]=\delta_{j k} . H$ is a Hermitian matrix which reads

$$
H=\Delta\left(\Omega_{-}, \Omega_{+}\right),
$$

where $\Omega_{-}$and $\Omega_{+} \in C^{n \times n}$ satisfying $\Omega_{-}=\Omega_{-}^{\dagger}$ and $\Omega_{+}=$ $\Omega_{+}^{T}$. The self-adjoint operator $\frac{1}{2} \breve{a}^{\dagger} H \breve{a}$ is the initial internal energy of the oscillators. With these parameters, $G$ on the joint system-field space can be written as

$$
\begin{aligned}
d \breve{a}(t) & =\left(-\frac{1}{2} C^{b} C-i \Psi H\right) \breve{a}(t) d t-C^{b} d \breve{B}_{\text {in }}(t) \\
d \breve{B}_{\text {out }}(t) & =C \breve{a}(t) d t+d \breve{B}_{\text {in }}(t), \quad \breve{a}(0)=\breve{a},
\end{aligned}
$$

in which $C=\Delta\left(C_{-}, C_{+}\right)$.

Remark 1: System (3) is called the annihilation-creation representation of linear quantum optical systems, which are parameterized by physical parameters like $C_{-}, C_{+}, \Omega_{-}$, and $\Omega_{+}$. Such parametrization is appealing in that it provides a convenient way for control design. This point will be elaborated in Sec. IV-B.

\section{Ideal Squeezers}

In quantum optical experiments, the boson field $b_{\text {in }}$ can be squeezed before they interact with quantum optical systems. Squeezing components can be approximated by Bogoliubov transformations. Let $S_{-}$and $S_{+} \in \mathbb{C}^{m \times m}$. Define $S=$ $\Delta\left(S_{-}, S_{+}\right)$. If $S$ satisfies

$$
S^{b} S=S S^{b}=I_{2 m}
$$

then $S$ is called a static Bogoliubov transformation. This paper focuses on a particular class of static Bogoliubov transformations. Let $\Sigma$ be a real diagonal matrix. Then it can be verified that

$$
S=\exp (\Delta(0, \Sigma))
$$

satisfies (4), hence it is a static Bogoliubov transformation. In this paper, such type of Bogoliubov transformations are called ideal squeezers, which have been widely used in quantum optics [6]. Clearly, $S$ is an identity matrix if $\Sigma$ is a zero matrix, that is, there is no squeezing.

Example 1: An ideal squeezer can be regarded as an idealization of a phase-shifted degenerate parametric amplifier (DPA). A DPA is an open oscillator that is able to produce squeezed output field. A model of a DPA is as follows ([6, page 220]):

$$
\begin{aligned}
d \breve{a}(t) & =-\frac{1}{2}\left[\begin{array}{cc}
\kappa & -\epsilon \\
-\epsilon & \kappa
\end{array}\right] \breve{a}(t) d t-\sqrt{\kappa} d \breve{B}_{\text {in }}(t) \\
d \breve{B}_{\text {out }}(t) & =\sqrt{\kappa} \breve{a}(t) d t+d \breve{B}_{\text {in }}(t) .
\end{aligned}
$$

For this system, $\Omega_{-}=0, \Omega_{+}=\frac{i \epsilon}{2}, C_{-}=\sqrt{\kappa}$, and $C_{+}=0$, $(\kappa>\epsilon)$. Therefore,

$$
A=-\frac{1}{2}\left[\begin{array}{cc}
\kappa & -\epsilon \\
-\epsilon & \kappa
\end{array}\right], C=\sqrt{\kappa} I, B=-\sqrt{\kappa}, D=I .
$$

Replace $\kappa$ and $\epsilon$ by $\frac{\kappa}{h}$ and $\frac{\epsilon}{h}$ respectively, where $h>0$. Define

$$
S=\left[\begin{array}{cc}
\frac{\kappa^{2}+\epsilon^{2}}{\kappa^{2}-\epsilon^{2}} & \frac{2 \kappa \epsilon}{\kappa^{2}-\epsilon^{2}} \\
\frac{2 \kappa \epsilon}{\kappa^{2}-\epsilon^{2}} & \frac{\kappa^{2}+\epsilon^{2}}{\kappa^{2}-\epsilon^{2}}
\end{array}\right]
$$

Clearly, $S^{\mathrm{b}} S=I$, that is, $S$ is indeed an ideal squeezer. It is easy to show that $D P A-(-S)$ is asymptotically stable for all $h>0$; moreover, when $h \rightarrow 0$. Finally, let

$$
r=\ln \frac{\kappa+\epsilon}{\kappa-\epsilon} .
$$

Then $S=\exp (\Delta(0, r))=\Delta(\cosh r, \sinh r)$. That is, an ideal squeezer is a limit of a sequence of DPAs with phase $\operatorname{shift}(\pi)$.

Remark 2: It is worth pointing out that the above convergence result does not depend on the specific choices of $\kappa$ and $\epsilon$ (however, $\kappa>\epsilon$ is assumed for the stability of the DPA.) 


\section{Open Quantum Optical Systems with Ideal Squeezers}

If the input field $b_{i n}(t)$ first pass through ideal squeezers $S$ prior to interacting with a collection of open quantum harmonic oscillators, then the composite system reads

$$
\begin{aligned}
d \breve{a}(t) & =A \breve{a}(t) d t+B S d \breve{B}_{\text {in }}(t), \quad \breve{a}(0)=\breve{a}, \\
d \breve{B}_{\text {out }}(t) & =C \breve{a}(t) d t+S d \breve{B}_{\text {in }}(t),
\end{aligned}
$$

in which the coefficient matrices are given by

$$
A=-\frac{1}{2} C^{b} C-i \Psi_{n} H, \quad B=-C^{b} .
$$

Using the notation in section II-B, the composite system can be represented by a triple $(S, L, H)$.

\section{E. Quadrature Representation}

So far, we have used the annihilation and creation operators $a_{j}$ and $a_{j}^{*}$ to represent oscillators systems, via the doubled-up notation $\breve{a}=\left[\begin{array}{ll}a^{T} & a^{\dagger}\end{array}\right]^{T}$. There is an alternative form, real-phase quadrature representation, in which all the operators are observables (self-adjoint operators) and all the corresponding matrices are real, not imaginary.

For example, consider the system in Fig. 1(a) with one oscillator interacting with one input field. Denote $M=$ $\frac{1}{\sqrt{2}}\left[\begin{array}{lll}1 & 1 ;-i & i\end{array}\right]$. Define operators $x=M \breve{a}, \tilde{b}_{\text {out }}=M \breve{b}_{\text {out }}$, and $\tilde{b}_{i n}=M \breve{b}_{\text {in }}$. Then $x, \tilde{b}_{\text {out }}, \tilde{b}_{\text {in }}$ are all self-adjoint operators, and all the corresponding matrices are real.

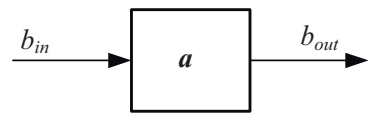

(a)

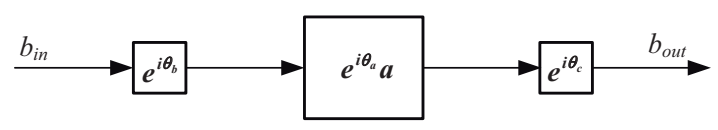

(b)

Fig. 1. Quadrature representation

Phase modulation is a technique which can be used to change phase of a light beam. Phase modulators are widely used in experimental quantum optics [1]. In this paper we show that systematic design of phase modulation can help to improve closed-loop control performance considerably. In this subsection, we introduce a new type of quadrature representation with built-in phase modulation. An example is also given to illustrate its usefulness in measurement-based feedback quantum control.

Let $P_{n}$ be a $2 n \times 2 n$ permutation matrix which transform a column vector $d=\left[d_{1}, \ldots, d_{2 n}\right]$ to $P_{n} d=$ $\left[d_{1}, d_{3}, d_{5}, \ldots, d_{2}, d_{4}, \ldots d_{2 n}\right]$. Let $M_{k}$ be a unitary matrix defined via

$$
M_{k}=\frac{1}{\sqrt{2}}\left[\begin{array}{cc}
e^{i \theta_{k}} & e^{-i \theta_{k}} \\
-i e^{i \theta_{k}} & i e^{-i \theta_{k}}
\end{array}\right],
$$

where $\theta_{k}$ is real. Introduce

$M_{a}=\operatorname{diag}\left(M_{1}, \ldots, M_{n}\right), M_{b}=\operatorname{diag}\left(M_{n+1}, \ldots, M_{n+m}\right)$,

$$
M_{c}=\operatorname{diag}\left(M_{n+m+1}, \ldots, M_{n+2 m}\right) .
$$

Then define the following transformation

$$
\Lambda_{a}=P_{n}^{T} M_{a} P_{n}, \quad \Lambda_{b}=P_{n}^{T} M_{b} P_{n}, \quad \Lambda_{c}=P_{n}^{T} M_{c} P_{n} .
$$

It can be readily verified that

$$
\Lambda_{j}^{\dagger} \Lambda_{j}=\Lambda_{j} \Lambda_{j}^{\dagger}=I, \quad j=a, b, c .
$$

Define a coordinate transform

$$
x=\Lambda_{a} \breve{a}, \quad \tilde{B}_{i n}=\Lambda_{b} \breve{B}_{\text {in }}, \quad \tilde{y}=\Lambda_{c} \breve{B}_{\text {out }} .
$$

Then in quadrature form, the system $G$ in Eq. (7) is converted to

$$
\begin{aligned}
d x(t) & =\tilde{A} x(t) d t+\tilde{B} \tilde{S} d \tilde{B}_{i n}(t), \quad x(0)=x, \\
d \tilde{y}(t) & =\tilde{C} x(t) d t+\Lambda_{c} \Lambda_{b}^{\dagger} \tilde{S} d \tilde{B}_{i n}(t)
\end{aligned}
$$

where

$$
\tilde{A}=\Lambda_{a} A \Lambda_{a}^{\dagger}, \tilde{B}=\Lambda_{a} B \Lambda_{b}^{\dagger}, \tilde{C}=\Lambda_{c} C \Lambda_{a}^{\dagger}, \tilde{S}=\Lambda_{b} S \Lambda_{b}^{\dagger} .
$$

Note that all entries of the matrices in this representation are real.

The above representation contains the real-phase quadrature representation as a special case. More specifically, it is the real-phase quadrature representation of the system from $b_{\text {in }}$ to $b_{\text {out }}$ in Fig. 1(b), not that in Fig. 1(a). That is, input, output and intra-cavity fields are implicitly assumed to be possibly phase-shifted in this new quadrature representation. This new quadrature representation enables us to choose suitable quadratures which yield the best (closed-loop) control performance.

Example 2: The following linear quantum plant is studied in $[17$, Sec. 8$]$ :

$$
\begin{aligned}
d x(t)= & {\left[\begin{array}{cc}
0 & \Delta \\
-\Delta & 0
\end{array}\right] x(t) d t+\left[\begin{array}{cc}
0 & 0 \\
0 & -2 \sqrt{k_{1}}
\end{array}\right] d \tilde{u}(t) } \\
& +\left[\begin{array}{cc}
0 & 0 \\
0 & -2 \sqrt{k_{2}}
\end{array}\right] d \tilde{B}_{i n, 1}(t) \\
& +\left[\begin{array}{cc}
0 & 0 \\
0 & -2 \sqrt{k_{3}}
\end{array}\right] d \tilde{B}_{i n, 2}(t) \\
d \tilde{y}(t)= & {\left[\begin{array}{cc}
2 \sqrt{k_{2}} & 0 \\
0 & 0
\end{array}\right] x(t) d t+d \tilde{B}_{i n, 1}(t) } \\
\tilde{z}(t)= & x(t)+\tilde{\beta}_{u}(t)
\end{aligned}
$$

where $\Delta=0.1, k_{1}=k_{2}=k_{3}=0.01, \tilde{\beta}_{u}$ is the signal part of $\tilde{u}$. This model corresponds to a quantum optics model characterized by

$$
\Omega_{-}=\Delta, \Omega_{+}=0, C_{-}=C_{+}=\left[\begin{array}{c}
\sqrt{k_{1}} \\
\sqrt{k_{2}} \\
\sqrt{k_{3}}
\end{array}\right], S=I .
$$

Clearly,

$$
\begin{gathered}
A=-i\left[\begin{array}{cc}
\Delta & 0 \\
0 & -\Delta
\end{array}\right], B_{l}=-\sqrt{\kappa_{l}}\left[\begin{array}{cc}
1 & -1 \\
-1 & 1
\end{array}\right], \\
C_{2}=\sqrt{\kappa_{2}}\left[\begin{array}{ll}
1 & 1 \\
1 & 1
\end{array}\right], l=1,2,3
\end{gathered}
$$


and the plant can be written as

$$
\begin{aligned}
d \breve{a} & =A \breve{a} d t+B_{1} d \breve{B}_{i n, 1}+B_{2} d \breve{B}_{i n, 2}+B_{3} d \breve{B}_{i n, 3} \\
d \breve{B}_{\text {out }, 2} & =C \breve{a} d t+d \breve{B}_{\text {in }, 2},
\end{aligned}
$$

Define a quadrature representation

$$
\begin{gathered}
x=\Lambda_{a} a, \tilde{u}=\Lambda_{b} \breve{B}_{i n, 1}, \tilde{w}_{1}=\Lambda \breve{B}_{\text {in }, 2}, \\
\tilde{w}_{2}=\Lambda \breve{B}_{\text {in }, 3}, \tilde{y}=\Lambda_{c} \breve{B}_{\text {out }, 2},
\end{gathered}
$$

where

$$
\begin{gathered}
\Lambda_{a}=\frac{1}{\sqrt{2}}\left[\begin{array}{cc}
e^{i \theta_{1}} & e^{-i \theta_{1}} \\
-i e^{i \theta_{2}} & i e^{-i \theta_{1}}
\end{array}\right], \Lambda_{b}=\frac{1}{\sqrt{2}}\left[\begin{array}{cc}
e^{i \theta_{2}} & e^{-i \theta_{2}} \\
-i e^{i \theta_{2}} & i e^{-i \theta_{2}}
\end{array}\right] . \\
\Lambda=\frac{1}{\sqrt{2}}\left[\begin{array}{cc}
1 & 1 \\
-i & i
\end{array}\right], \Lambda_{c}=\frac{1}{\sqrt{2}}\left[\begin{array}{cc}
e^{i \theta_{3}} & e^{-i \theta_{3}} \\
-i e^{i \theta_{3}} & i e^{-i \theta_{3}}
\end{array}\right] .
\end{gathered}
$$

Then, in quadrature representation the system is,

$$
\begin{aligned}
d x & =\tilde{A} x d t+2 \sqrt{\kappa_{1}} \tilde{B}_{1} d \tilde{u}+\tilde{B}_{2} d \tilde{w}_{1}+\tilde{B}_{3} d \tilde{w}_{2}, \\
\tilde{y} & =\tilde{C}_{2} x d t+\tilde{D} d \tilde{w}_{1} \\
\tilde{z} & =x+\tilde{\beta}_{u}
\end{aligned}
$$

where $\tilde{\beta}_{u}$ is the signal part of $\tilde{u}$, and

$$
\begin{gathered}
\tilde{A}=\left[\begin{array}{cc}
0 & \Delta \\
-\Delta & 0
\end{array}\right], \tilde{B}_{l}=\left[\begin{array}{cc}
0 & 0 \\
0 & -2 \sqrt{\kappa_{l}}
\end{array}\right], \\
\tilde{B}_{1}=2 \sqrt{\kappa_{1}}\left[\begin{array}{cc}
-\sin \left(\theta_{1}\right) \sin \left(\theta_{2}\right) & \sin \left(\theta_{1}\right) \cos \left(\theta_{2}\right) \\
\cos \left(\theta_{1}\right) \sin \left(\theta_{2}\right) & -\cos \left(\theta_{1}\right) \cos \left(\theta_{2}\right)
\end{array}\right], \\
\tilde{C}_{2}=2 \sqrt{\kappa_{2}}\left[\begin{array}{cc}
\cos \left(\theta_{3}\right) \cos \left(\theta_{1}\right) & \cos \left(\theta_{3}\right) \sin \left(\theta_{1}\right) \\
\sin \left(\theta_{3}\right) \cos \left(\theta_{1}\right) & \sin \left(\theta_{3}\right) \sin \left(\theta_{1}\right)
\end{array}\right], \\
\tilde{D}=\left[\begin{array}{cc}
\cos \left(\theta_{3}\right) & -\sin \left(\theta_{3}\right) \\
\sin \left(\theta_{3}\right) & \cos \left(\theta_{3}\right)
\end{array}\right], l=2,3 .
\end{gathered}
$$

To compare with results in [17, Sec. 8.2], only the first element of $\tilde{y}$ is used. When $\theta_{1}=\theta_{2}=\theta_{3}=0$, the system reduces to Eq. (38) in [17]. It has been shown in Sec. 8.2 of [17] that a measurement-based feedback controller yields a closed-loop LQG index 4.8468. In what follows we study several other cases.

(i). When $\theta_{1}=\theta_{2}=0$ and $\theta_{3} \neq 0$, we have homodyne detection. Numerical study shows that $\theta_{3}=0$ gives rise to an LQG performance 4.8468 . That is, there is no improvement.

(ii). When $\theta_{1}=0, \theta_{2}=-0.5294$, and $\theta_{3}=-0.5498$, the LQG performance is 4.0551 , which is not only better than 4.8468 , but also better than 4.1793 (the best coherent LQG control performance obtained in [17]).

(iii). When $\theta_{1}=0.5341$ and $\theta_{2}=\theta_{3}=0$, the LQG performance is 3.3734 .

(iv). When $\theta_{1}=0.5500, \theta_{2}=4.0157$, and $\theta_{3}=0.2985$, the LQG performance is found to be 3.2515 .

This example demonstrates that measurement-based feedback plus phase modulation can yield better LQG control performance.

\section{F. Fundamental Relations}

In this subsection a characterization of matrices defined in Eq. (11) is presented. Moreover, some fundamental relations are derived.

Define a symmetric matrix $\mathbf{R} \in \mathbb{R}^{2 n \times 2 n}$ and a coupling matrix $\Lambda \in \mathbb{C}^{m \times 2 n}$ to be $\mathbf{R}:=\Lambda_{a} H \Lambda_{a}^{\dagger}$ and $\boldsymbol{\Lambda}:=$ $\left[\begin{array}{ll}C_{-} & C_{+}\end{array}\right] \Lambda_{a}^{\dagger}$ respectively, then an alternative form of system matrices of (11) can be derived.

Proposition 1: System matrices defined in (11) satisfy the following relations:

$$
\begin{aligned}
\tilde{A} & =J_{n}\left\{\operatorname{Im}\left\{\boldsymbol{\Lambda}^{\dagger} \boldsymbol{\Lambda}\right\}+\mathbf{R}\right\}, \\
\tilde{B} & =-i J_{n}\left[\boldsymbol{\Lambda}^{\dagger} \boldsymbol{\Lambda}^{T}\right] \Psi_{m} \Lambda_{b}^{\dagger}, \\
\tilde{C} & =\Lambda_{c}\left[\begin{array}{c}
\boldsymbol{\Lambda} \\
\boldsymbol{\Lambda}^{\#}
\end{array}\right] .
\end{aligned}
$$

Remark 3: Characterization similar to Eqs. (14)-(16) has been used in Definition 3.1 in [12] for the real-phase quadrature representation. Proposition 1 shows these relations can be obtained based on the relation between annihilationcreation representation and quadrature representation.

The so-called physical realizability has been investigated in [12], [16], which exemplifies some fundamental relations that a quantum system must obey. Unfortunately its derivation is pretty much mathematically involved. Interested reader may check the proofs of Theorems 2.1 and 3.4 of [12] where a construction procedure is given. In the following, starting from the annihilation-creation representation we show that a similar characterization of physical realizability can be derived rather simply.

Theorem 1: The real matrices $\tilde{A}, \tilde{B}$, and $\tilde{C}$ in Eq. (11) satisfy the following equations:

$$
\begin{gathered}
\tilde{A} J_{n}+J_{n} \tilde{A}^{\dagger}+\tilde{B} J_{m} \tilde{B}^{\dagger}=0, \\
\tilde{B}=-i J_{n} \tilde{C}^{\dagger}\left(\Lambda_{c} \Psi_{m} \Lambda_{b}^{\dagger}\right) .
\end{gathered}
$$

\section{Closed Loop And Performance Convergence}

This section presents the setup of the closed-loop quantum systems.

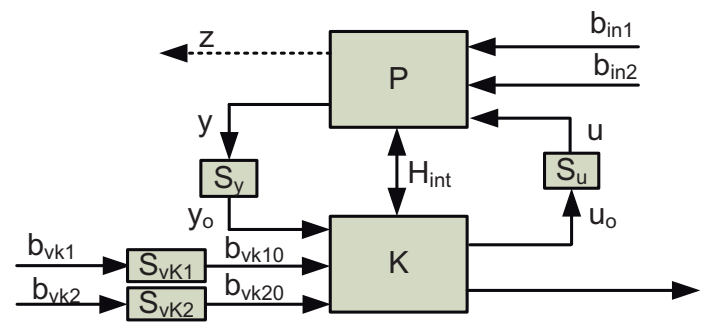

Fig. 2. Coherent feedback control

Consider the closed-loop system as shown in Fig. 2, where $P$ is a quantum plant and $K$ is a coherent feedback controller to be designed. Here, $b_{v}$ and $b_{i n}$ are quantum noises, $b_{v}$ is in vacuum state, while $b_{i n}$ may have finite energy. $y$ is plant output (output field channel), $u$ is control input (input field channel), $u_{o}$ is the output of the controller $K . z$ is reference 
output (namely, a performance variable, which may not be a physical variable, so is designated by a dotted line), $b_{v_{k 1}}$ and $b_{v_{k 2}}$ are quantum noises to the controller $K$ in vacuum state.

In terms of quadrature representation introduced in Sec. II-E, the quantum plant $P$ is described by

$$
\begin{aligned}
d x(t) & =\tilde{A} x(t) d t+\tilde{B}_{0} d \tilde{B}_{v}(t)+\tilde{B}_{1} d \tilde{B}_{i n}(t)+\tilde{B}_{2} d \tilde{u}(t), \\
d \tilde{z}(t) & =\tilde{C}_{1} x(t) d t+\tilde{D}_{12} d \tilde{u}(t)+\tilde{D}_{w} d \tilde{B}_{i n}(t) \\
d \tilde{y}(t) & =\tilde{C}_{2} x(t) d t+\tilde{D}_{20} d \tilde{B}_{v}(t)+\tilde{D}_{21} d \tilde{B}_{i n}(t)
\end{aligned}
$$

and the controller by

$$
\begin{aligned}
d \xi(t)= & \tilde{A}_{K} \xi(t) d t+\tilde{B}_{K 1} d \tilde{B}_{v_{K 10}}(t) \\
& +\tilde{B}_{K 2} d \tilde{B}_{v_{K 20}}(t)+\tilde{B}_{K} d \tilde{y}_{o}(t), \\
d \tilde{u}_{o}(t)= & \tilde{C}_{K} \xi(t) d t+\tilde{D}_{K} d \tilde{B}_{v_{K 10}}(t) .
\end{aligned}
$$

$H_{\text {int }}$ in Fig. 2 designates the direct coupling between $P$ and $K$. Direct coupling is a physical mechanism by which a quantum plant and its quantum controller can directly exchange energy, without necessarily through field connections (cf. [13, Sec. II-C and Fig. 4], [22, Sec. II-B]). Direct coupling can be implemented via nonlinear optical devices like crystals [19, Fig. 1].

Define matrices

$$
\begin{aligned}
\tilde{A}_{c l} & =\left[\begin{array}{cc}
\tilde{A} & \tilde{B}_{2} \tilde{S}_{u} \tilde{C}_{K}+\tilde{B}_{12} \\
\tilde{B}_{K} \tilde{S}_{y} \tilde{C}_{2}+\tilde{B}_{21} & \tilde{A}_{K}
\end{array}\right], \\
\tilde{B}_{c l} & =\left[\begin{array}{c}
\tilde{B}_{1} \\
\tilde{B}_{K} \tilde{S}_{y} \tilde{D}_{21}
\end{array}\right], \tilde{C}_{c l}=\left[\begin{array}{ll}
\tilde{C}_{1} & \tilde{D}_{12} \tilde{S}_{u} \tilde{C}_{K}
\end{array}\right] \\
\tilde{G}_{c l} & =\left[\begin{array}{ccc}
\tilde{B}_{0} & \tilde{B}_{2} \tilde{S}_{u} \tilde{D}_{K} \tilde{S}_{v K 1} & 0 \\
\tilde{B}_{K} \tilde{S}_{y} \tilde{D}_{20} & \tilde{B}_{K 1} \tilde{S}_{v K 1} & \tilde{B}_{K 2} \tilde{S}_{v K 2}
\end{array}\right], \\
\tilde{H}_{c l} & =\left[\begin{array}{ccc}
0 & \tilde{D}_{12} \tilde{S}_{u} \tilde{D}_{K} \tilde{S}_{v K 1} & 0
\end{array}\right],
\end{aligned}
$$

where $\tilde{B}_{12}$ and $\tilde{B}_{21}$ are for direct coupling, satisfying $\tilde{B}_{21}=$ $J_{n} \tilde{B}_{12}^{T} J_{n}$ with $\tilde{B}_{12}$ being an arbitrary real-valued matrix [22]. The closed-loop system with a built-in direct coupling in the quadrature representation is given by

$$
\begin{gathered}
{\left[\begin{array}{c}
d x(t) \\
d \xi(t)
\end{array}\right]=\tilde{A}_{c l}\left[\begin{array}{l}
x(t) \\
\xi(t)
\end{array}\right] d t+\tilde{B}_{c l} d \tilde{B}_{i n}(t)+\tilde{G}_{c l}\left[\begin{array}{c}
d \tilde{B}_{v}(t) \\
d \tilde{B}_{v_{K 1}}(t) \\
d \tilde{B}_{v_{K} 2}(t)
\end{array}\right.} \\
d \tilde{z}(t)=\tilde{C}_{c l}\left[\begin{array}{c}
x(t) \\
\xi(t)
\end{array}\right] d t+\tilde{D}_{w} d \tilde{B}_{i n}(t) \tilde{H}_{c l}\left[\begin{array}{c}
d \tilde{B}_{v}(t) \\
d \tilde{B}_{v_{K 1}}(t) \\
d \tilde{B}_{v_{K} 2}(t)
\end{array}\right]
\end{gathered}
$$

\section{LQG SYNTHESIS}

\section{A. Set-up}

In this subsection, we formulate the coherent quantum LQG control problem.

The following assumptions:

A1. $\tilde{D}_{w}$ in the plant (19) is zero.

A2. There is no additional noise input $b_{v}(t)$ in the plant (19).

A4. The performance variable is $\tilde{z}(t)=\tilde{C}_{1} x(t)+$ $\tilde{D}_{12} \tilde{\beta}_{u_{o}}(t)$, where $\tilde{\beta}_{u_{o}}(t)$ is the signal part of $u_{o}(t)$.
A controller of the form (20) is sought to minimize a quadratic performance index

$$
J_{\infty}=\lim \sup _{t \rightarrow \infty} \frac{1}{t} \int_{0}^{t}\left\langle z^{T}(s) z(s)\right\rangle d s .
$$

Suppose that $\tilde{A}_{c l}$ in (21) is Hurwitz, then a positive definite matrix $\mathcal{P}_{L Q G}$ is the (unique) solution to the following Lyapunov equation

$$
\tilde{A}_{c l} \mathcal{P}_{L Q G}+\mathcal{P}_{L Q G} \tilde{A}_{c l}^{T}+\left[\begin{array}{ll}
\tilde{B}_{c l} & \tilde{G}_{c l}
\end{array}\right]\left[\begin{array}{cc}
\tilde{B}_{c l} & \tilde{G}_{c l}
\end{array}\right]^{T}=0 .
$$

Note that $\tilde{G}_{c l}=\left[\begin{array}{lll}\tilde{B}_{2} \tilde{D}_{K} & 0 ; \tilde{B}_{K 1} & \tilde{B}_{K 2}\end{array}\right]$ since there is no the additional noise input $b_{v}(t)$. It is shown in [17] that $J_{\infty}$ in (22) can be computed via

$$
J_{\infty}=\operatorname{Tr}\left(\tilde{C}_{c l} \mathcal{P}_{L Q G} \tilde{C}_{c l}^{T}\right)
$$

based on which a quantum LQG control problem can be formulated and solved.

\section{B. Controller Parameterization and a Numerical Optimiza-} tion Approach - without Phase Modulation

According to Section II-B we see the controller can be parameterized by $C_{-}, C_{+}, \Omega_{-}, \Omega_{+}$, from Section II-C we see that each ideal squeezer can be parametrized by a real number; and direct coupling and phase modulation involve just a couple of parameters. Moreover, with such parameterization physical realizability is naturally satisfied. This enlightens us to seek for controller design methods via optimization over these parameters. Inspired by the study in [3], in this and the next sections we investigate a new optimization approach on the basis of controller parameterization and a two-stage optimization algorithm.

In the following we first present controller parameterization. The coherent feedback controller to be constructed is in the form of Eq. (20), that is, a quantum harmonic oscillator interacting with three input fields. For simplicity, assume both $C_{-}$and $C_{+}$are real matrices. According to Sec. II$\mathrm{B}$, there are 9 parameters to be optimized. Direct coupling requires 4 parameters, each of the four squeezers needs one parameter respectively. Therefore, we will optimize over 17 parameters simultaneously.

Based on this parametrization, a two-stage optimization is constructed: Stage one is a genetic algorithm (GA) which searches for an initial solution [7]; Stage two utilizes this initial solution to conduct local search to find an optimal solution. In what follows the algorithm is analyzed in more detail.

In Stage one, a genetic algorithm will optimize over the cost function (24) subject to the following constraints.

1) The closed-loop matrix $\tilde{A}_{c l}$ is Hurwitz.

2) The Lyapunov equation (23) has a (unique) positive definite solution $\mathcal{P}_{L Q G}$.

Note that physical realizability is satisfied naturally by means of parametrization. The genetic algorithm performs a global search over the parameter space to find a minimal solution. 
In Stage two, a local search is performed around the minimal solution derived from Stage one. Around this solution, the closed-loop matrix $\tilde{A}_{c l}$ is always Hurwitz, hence the optimization problem boils down to one minimizing the cost function (24) subject to the constraint (23). Here, the cost function and constraints are all continuously differentiable. Consequently, this optimization problem can be solved by means of many standard optimization algorithms.

The preceding numerical algorithm turns out to be very effective. It provides the following solution:

$$
\begin{gathered}
C_{-}=\left[\begin{array}{c}
0.0566 \\
0.2370 \\
33.1525
\end{array}\right], C_{+}=\left[\begin{array}{c}
-0.0566 \\
0.2212 \\
0.3441
\end{array}\right], \\
\Omega_{-}=0.0205, \Omega_{+}=2.9041-3.4880 i .
\end{gathered}
$$

Therefore, the matrices of controller $K$ are

$$
\begin{gathered}
\tilde{A}_{K}=\left[\begin{array}{cc}
-552.9770 & -2.8836 \\
-2.9245 & -546.0009
\end{array}\right], \tilde{B}_{K 1}=\left[\begin{array}{cc}
-0.1131 & 0 \\
0 & 0
\end{array}\right] \\
\tilde{B}_{K 2}=\left[\begin{array}{cc}
-0.0158 & 0 \\
0 & -0.4582
\end{array}\right], \tilde{C}_{K}=\left[\begin{array}{cc}
0 & 0 \\
0 & 0.1131
\end{array}\right] \\
\tilde{B}_{K}=\left[\begin{array}{cc}
-32.8084 & 0 \\
0 & -33.4966
\end{array}\right], \quad \tilde{D}_{K}=I,
\end{gathered}
$$

The matrices for direct coupling are

$$
\begin{gathered}
\tilde{B}_{12}=\left[\begin{array}{cc}
234.0754 & 322.5037 \\
-207.1394 & 351.9539
\end{array}\right], \\
\tilde{B}_{21}=\left[\begin{array}{cc}
-351.9539 & 322.5037 \\
-207.1394 & -234.0754
\end{array}\right] .
\end{gathered}
$$

Finally ideal squeezers are

$$
\begin{gathered}
\tilde{S}_{u}=\left[\begin{array}{cc}
539.4476 & 0 \\
0 & 0.0019
\end{array}\right], \tilde{S}_{v_{K 1}}=\left[\begin{array}{cc}
0.0839 & 0 \\
0 & 11.9174
\end{array}\right] \\
\tilde{S}_{v_{K 2}}=\left[\begin{array}{cc}
6.7026 & 0 \\
0 & 0.1492
\end{array}\right], \tilde{S}_{y}=\left[\begin{array}{cc}
1.2607 & 0 \\
0 & 0.7932
\end{array}\right] .
\end{gathered}
$$

The resulting LQG performance is 2.0001.

\section{CONCLUSION}

Recent years have seen a considerable amount of work on quantum networks theory, e.g., system interconnection [21], [8], [9], [10], $H^{\infty}$ control [12], LQG control [17], synthesis [16], dissipation theory and direct coupling [13], [22], laboratory demonstrations [14], among others. This paper fits into this general picture by presenting how to use phase modulation and squeezing in control design for linear quantum optical feedback networks. In addition, the controller parameterization and two-stage optimization procedure may be useful in the optimal design of quantum optical networks.

\section{REFERENCES}

[1] H.A. Bachor and T.C. Ralph, A Guide to Experiments in Quantum Optics, 2nd ed, Weinheim, Germany: Wiley-VCH, 2004.

[2] V.P. Belavkin, "On the theory of controlling observiable quantum systems," Automation and Remote Control, vol. 44, no. 2, pp. 178188, 1983.

[3] D.U. Campos-Delgado and K. Zhou, "Mixed $L_{1} / H_{2} / H_{\infty}$ control design: numerical optimization approaches, " Int. J. Control, vol. 76, no. 7, pp. 687-697, 2003.

[4] A. C. Doherty and K. Jacobs, "Feedback-control of quantum systems using continuous state-estimation," Phys. Rev. A, vol. 60, pp. 2700$2711,1999$.

[5] D. Dong D and I R. Petersen. "Quantum control theory and applications: A survey," IET Control Theory \& Applications, vol. 4, no. 12 , pp. 2651-2671, 2010.

[6] C. Gardiner and P. Zoller, Quantum Noise, Springer, Berlin, 2004.

[7] D.E. Goldberg, Genetic Algorithms in Search, Optimization \& Machine Learning, Addison-Wesley Longman Publishing Co., Inc. Boston, MA, USA, 1989.

[8] J.E. Gough and M.R. James, "Quantum feedback networks: Hamiltonian formulation," Commun. Math. Phys., vol. 287, pp. 1109-1132, 2009.

[9] J.E. Gough and M.R. James, "The series product and its application to quantum feedforward and feedback networks," IEEE Trans. Automatic Control, vol. 54, no.11, pp. 2530-2544, 2009.

[10] J.E. Gough, M.R. James and H.I. Nurdin, "Squeezing components in linear quantum feedback networks," Phys. Rev. A, vol. 81, 023804, 2010.

[11] R.L. Hudson and K.R. Parthasarathy, "Quantum Ito's formula and stochastic evolutions," Commun. Math. Phys., vol. 93, pp. 301-323, 1984.

[12] M.R. James, H.I. Nurdin, and I.R. Petersen, " $H^{\infty}$ control of linear quantum stochastic systems," IEEE Trans. Automat. Control, vol. 53, no. 8, pp. 1787-1803, 2008.

[13] M.R. James and J.E. Gough. "Quantum dissipative systems and feedback control design by interconnection," IEEE Trans. Automat. Control, vol. 55, no. 8, pp. 1806-1821, 2010.

[14] H. Mabuchi, "Coherent-feedback quantum control with a dynamic compensator," Phys. Rev. A, vol. 78, 032323, 2008.

[15] G.J. Milburn and H.W. Wiseman, Quantum Measurement and Control, Cambridge University Press, 2009.

[16] H.I. Nurdin, M.R. James, and A.C. Doherty, "Network synthesis of linear dynamical quantum stochastic systems," SIAM J. Control and Optim., vol. 48, no. 4, pp. 2686-2718, 2009.

[17] H.I. Nurdin, M.R. James, and I.R. Petersen, "Coherent quantum LQG control," Automatica, vol. 45, pp. 1837-1846, 2009.

[18] R. van Handel, J.K. Stockton, and H. Mabuchi, 'Feedback control of quantum state reduction," IEEE Trans. Automat. Contr., vol. 50, no. 6, pp. 768-780, 2005.

[19] H.M. Wiseman and G.J. Milburn, "All-optical versus electro-optical quantum-limited feedback," Phys. Rev. A, vol. 49, pp. 4110-4125, 1994.

[20] H.M. Wiseman, S. Mancini, and J. Wang, "Bayesian feedback versus Markovian feedback in a two-level atom," Phys. Rev. A, vol. 66, 013807, 2002

[21] M. Yanagisawa and H. Kimura, "Transfer function approach to quantum control-part II: control concepts and applications,” IEEE Trans. Automat. Contr., vol. 48, pp. 2107-2132, 2003.

[22] G. Zhang and M.R. James, "Direct and indirect couplings in coherent feedback control of linear quantum systems," IEEE Trans. Automat. Contr., accepted, 2010 (arXiv:1010.4369v1 (quant-ph)). 